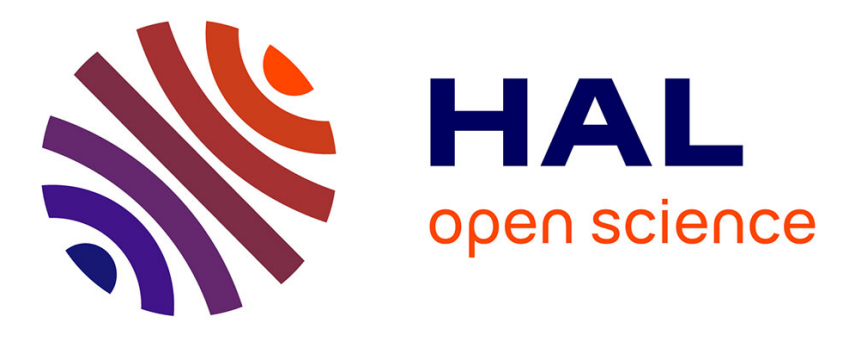

\title{
La France et l'Allemagne au cour de l'Europe industrielle en construction: des ententes privées à la CECA
}

Françoise Berger

\section{- To cite this version:}

Françoise Berger. La France et l'Allemagne au cœur de l'Europe industrielle en construction: des ententes privées à la CECA. Matériaux pour l'histoire de notre temps, 1997, Historiens d'hier et industrialisation en Europe occidentale de 1880 à la fin des années 1960, 47, pp.37-46. 10.3406/mat.1997.404264. halshs-00147425

\section{HAL Id: halshs-00147425 \\ https://shs.hal.science/halshs-00147425}

Submitted on 17 May 2007

HAL is a multi-disciplinary open access archive for the deposit and dissemination of scientific research documents, whether they are published or not. The documents may come from teaching and research institutions in France or abroad, or from public or private research centers.
L'archive ouverte pluridisciplinaire HAL, est destinée au dépôt et à la diffusion de documents scientifiques de niveau recherche, publiés ou non, émanant des établissements d'enseignement et de recherche français ou étrangers, des laboratoires publics ou privés.

\section{(ㅇ)(1) $\$$}

Distributed under a Creative Commons Attribution - NonCommercial - NoDerivatives| 4.0 


\section{La France et l'Allemagne au cour de l'Europe industrielle en construction: des ententes privées à la CECA.}

Françoise Berger

Le choix de textes qui suit à pour objectif de faire saisir les évolutions qui se sont produites en Europe dans l'idée de coopération économique internationale, en particulier industrielle. L'axe franco-allemand a été le pivot de la construction européenne de l'après-guerre qui s'est concrétisée en premier lieu par la signature du Traité de la Communauté Européenne du Charbon et de l'Acier (1951). Mais il avait déjà été celui des rapprochements industriels par secteur, à partir $d u$ milieu des années vingt, avec l'élaboration de divers cartels d'abord européens, puis internationaux, dans lesquels les deux pays occupaient une place prépondérante, en particulier au sein de l'Entente Internationale de l'Acier (1926 et 1932).

La CGPF et les ententes internationales au temps de la crise (1932)

Le "Bulletin Quotidien", dont est extrait cet article, a été créé dans les années vingt par André François-Poncet, pour l'information des industriels, et particulièrement des membres $d u$ Comité des Forges. Il reflète donc l'opinion des groupes dirigeants de l'industrie française. En cette fin d'année 1932 sont sur le point d'aboutir les négociations pour la reconstitution de l'Entente Internationale de l'Acier. Ce cartel européen avait été constitué en 1926 autour des sidérurgistes français, allemands, luxembourgeois et belges. La crise l'avait rapidement mis à bas, mais avec l'effondrement des prix, les producteurs relancent très vite des négociations. Celles-ci s'éternisent un peu en raison du manque de cohésion du groupe belge qui a du mal à discipliner de trop nombreux " outsiders " qui profitent de la perturbation due à la crise pour gagner des parts de marché par la pratique outrancière du dumping. La nouvelle Entente Internationale de l'Acier entre finalement en vigueur dès janvier 1933. L'article présente de façon complète les avantages de ces ententes, d'initiative privée, tels qu'ils sont perçus à cette époque, et leur utilité relative selon la conjoncture. La CGPF appelle de ses voux un soutien plus ouvert et plus efficace des principaux gouvernements européens à la formation d'ententes sectorielle tout en souhaitant que ces actions privées restent soutenues et complétées par les mesures publiques de soutien à la production industrielle.

\section{Les ententes et l'organisation de la production.}

M. Duchemin, présidant hier la section de l'organisation nationale et internationale de la production au Congrès des industries et commerces d'exportation, définissait le caractère essentiel de la crise actuelle en disant, d'après la vieille formule de J.B.Say, qu'il y avait moins surproduction que mauvaise répartition des produits par suite de l'anarchie des conditions de la production, observation de bon sens que l'analyse de la vie économique confirme en tous domaines. Pour remédier à cette anarchie, beaucoup de solutions ont été ou théoriquement préconisées, ou même pratiquement tentées. Parmi elles, les ententes nationales et internationales ont fait naître les plus vives espérances. La conférence économique de Genève, en 1927, les avait déjà mises à son ordre $\mathrm{du}$ jour. Le plan constructif du gouvernement français, présenté en mai 1931, au lendemain du protocole douanier austro-allemand, considérait la généralisation des ententes comme l'un des moyens les plus féconds de surmonter la dépression économique mondiale dont souffraient plus particulièrement les États de l'Europe centrale. L'accentuation de la crise donne une vigueur nouvelle aux efforts tentés pour une rationalisation de la production et des échanges par le moyen d'ententes nationales ou internationales à substituer à l'individualisme anarchique ou à l'intervention trop prononcée de l'État. Il est donc opportun de rechercher, à la lumière de l'expérience déjà acquise, quels ont été les effets de ces organisations économiques sur l'équilibre des marchés et les prix 
des produits, et dans quelle mesure elles peuvent utilement contribuer au redressement de la situation présente.

\section{L'évolution des ententes.}

Espérant que les ententes finiront, à leur tour, par exercer leur action régulatrice sur les conditions économiques, M. Gounod, vice-président délégué de l'Union des industries chimiques a montré, en premier lieu, que leur création avait été la conséquence des circonstances contemporaines. Les principales causes du déséquilibre qui s'est accentué au cours des dernières années entre la production et la consommation ont été les découvertes scientifiques et les progrès de machinisme transformant la base technique et financière des entreprises, la concurrence pour la recherche soit des matières premières ou des capitaux à bas prix, soit des débouchés.

Dans l'ordre industriel, les ententes se sont ainsi proposées des fins extrêmement différentes, telles: l'échange de brevets et de procédés, l'approvisionnement et la répartition des matières premières - notamment combustible, sources diverses d'énergie, ou minerais. Dans l'ordre commercial, elles ont visé tantôt la détermination des prix, tantôt la répartition des marchés. Si l'on recherche les moments propices ou défavorables, on constate, démontre M. Gounod, que:

1) Les périodes de prospérité, correspondant à un état de déséquilibre production-consommation, et caractérisées par une production inférieure à la consommation, sont peu favorables à la formation des ententes. Au cours de ces périodes, il ne s'en crée guère de nouvelles, celles qui existent déjà paraissent subir de graves difficultés, entravant leur bon fonctionnement; plusieurs d'entre elles se dissolvent même.

2) $\mathrm{Au}$ cours des périodes de dépression, le déséquilibre production-consommation se caractérise par un excédent de la production par rapport à la consommation. La conjoncture est défavorable aux industriels, mais les moins atteints se refuseront souvent, devant une consommation rétrécie, à lier leur sort à celui de leurs concurrents plus affaiblis. En fait, au cours de la présente crise, beaucoup d'ententes se sont dissoutes, ou ont du moins traversé des difficultés graves comme le prouvent les exemples suivants : entente du cuivre, dissoute en juillet 1932; du soufre, dissoute à la même date; difficultés de l'entente du pétrole.

3) Les périodes d'équilibre relatif, qui ne comportent ni prospérité extrême, ni dépression excessive, paraissent incliner davantage à la formation des ententes. En ce cas, en effet, on ne trouve aucun des facteurs qui contribuaient, dans les hypothèses précédentes, à s'opposer à leur formation. Tout semble au contraire les inciter à se constituer; l'allure assez bonne, mais sans excès des affaires, des difficultés relatives pour le placement d'une marchandise que l'on ne se dispute pas encore, la production et la consommation étant à peu près équilibrées. Chacun apporte à l'entente une part égale de chances et de risques. Aussi enregistre-t-on la formation des ententes du soufre et de l'aluminium en 1923; de l'acier, du cuivre, de la potasse, des rails, des colles d'os, du linoléum en 1926; du pétrole en 1927. Sans doute ne manquera-t-on pas de mettre en doute l'efficacité de ces ententes qui n'ont pu prévenir la crise présente. Peut-être n'est-il pas interdit de supposer que ses effets eussent été plus profonds encore sans les tentatives de rationalisation qui l'ont précédée. En tout cas, on peut tirer, pour l'avenir, les enseignements des défauts constatés dans la plupart des ententes afin de permettre à celles qui pourraient être constituées demain de remplir avec moins de risques le rôle d'adaptation entre l'offre et la demande qu'on attend d'elles.

\section{Les avantages des ententes.}

Ce rôle, M. Lambert-Ribot, délégué-général du Comité des Forges, l'a défini en analysant les conséquences de la réglementation de la concurrence: d'une part, sur les prix de vente, d'autre part, sur les prix de revient. En ce qui concerne les prix de vente, l'entente maintient leur stabilité pendant des périodes de 10 mois à 2 ans (pour les comptoirs intérieurs sidérurgiques), aussi utile aux producteurs qu'aux consommateurs, sans exclure d'ailleurs la possibilité d'une adaptation rapide pendant les périodes de crise; elle tend à uniformiser les barèmes, avantage particulièrement précieux sur le terrain international, en supprimant les systèmes de subventions, qui faussent toute notion de prix de revient, la politique ruineuse du dumping, qui vicie les relations internationales et les ventes à perte à l'exportation qui, telles qu'elles sont pratiquées actuellement, constituent une absurdité économique; elle permet l'établissement de prix rationnels échelonnés logiquement selon les frais réels de fabrication; enfin, l'entente assure aux consommateurs, malgré les croyances et, parfois, les apparences contraires, des prix modérés, l'exagération des cours entraînant le plus souvent (le cas du cuivre est caractéristique) des ruptures ruineuses. En ce qui concerne les prix de revient, les ententes permettent un abaissement des frais généraux par une meilleure utilisation des installations existantes et une diminution des transports grâce à une répartition logique des commandes, une diminution des frais commerciaux, des frais d'études ou de recherches.

L'énumération de ces avantages, dont profitent 
autant l'industrie que le consommateur, permet de regretter que certaines ententes ne puissent être conclues par la faute de rares dissidents. Sans doute conviendrait-il, conclut Lambert-Ribot, "que les gouvernements reconnaissent, à l'occasion de la prochaine conférence économique mondiale, que la seule voie rationnelle pour la réorganisation des marchés est la conclusion ententes internationales; qu'ils l'affirment hautement et se déclarent disposés à prendre éventuellement les mesures nécessaires pour amener leurs ressortissants à s'organiser afin de pouvoir conclure des accords internationaux".

\section{Les ententes et la politique tarifaire}

Cette collaboration privée entre les producteurs pourrait-elle se substituer éventuellement à l'action régulatrice des échanges que les pouvoirs publics exercent par l'intermédiaire des droits de douane, des contingents et autres mesures administratives susceptibles de compenser les inégalités naturelles ou fiscales des conditions de la production? M. de Lavergne, délégué de la Confédération générale de la production française, dans son rapport sur cette question ne le pense pas.

Dans certains cas, les industries se sont entendues pour fixer les conditions de prix auxquelles leurs produits peuvent être offerts sur certains marchés ou, même, comme les industries lainières récemment, les taux des droits de douane des divers pays participants. il semble que, dans ces hypothèses, le maintien des droits de douane ne se justifie pas ou s'explique seulement par des motifs fiscaux. Cependant, même dans le cas d'ententes très complètes, les plus favorables à l'abaissement des tarifs, ceux-ci ne pourraient être abandonnés sans délai: d'une part, leur expression serait nuisible à l'existence même des ententes, car elle inciterait les participants les plus favorisés à une dénonciation qui leur rendrait leur liberté de vente; d'autre part, elles apporteraient un avantage injustifié soit aux industries dissidentes des pays participants qui auraient refusé d'adhérer à l'entente, soit à celles des pays tiers.

Le maintien des droits de douane, qui pourrait d'ailleurs être atténué par un de ces systèmes de remboursement ou de compensation dont M. Le Troquer, sénateur et président du Comité international de l'Union douanière européenne a exposé les diverses modalités de réalisation, est donc une sauvegarde nécessaire pour les industries isolées comme pour les ententes elles-mêmes tant qu'existeront les pratiques de dumping et de concurrence déloyale et que le commerce n'aura pas retrouvé le fair-play d'un marché assaini. Les ententes privées ne peuvent donc pas remplacer l'action gouvernementale sur la régulation des échanges, mais la compléter ou la préparer.
" Les gouvernements, concluait M. de Lavergne doivent cesser d'avoir la crainte des ententes nationales et internationales. Ils doivent reconnaître que celles-ci sont nécessaires pour assurer l'équilibre économique et éviter des guerres commerciales néfastes à la bonne harmonie des peuples. Ils doivent d'ailleurs se rendre compte que par le jeu des droits de douane, sinon par la législation intérieure, ils sont suffisamment armés pour s'opposer dans l'intérêt des consommateurs aux abus qui pourraient résulter d'ententes dirigées vers la réalisation de bénéfices excessifs. Forts de cette constatation, ils pourront ainsi apporter l'appui de la puissance publique à la constitution et au maintien d'ententes qui peuvent concourir, dans un certain nombre de cas, à une politique de désarmement douanier".

Telle semble être la conclusion que l'expérience des dernières années permet de formuler sur les ententes : elles prennent place parmi les moyens de collaboration et d'organisation rationnelle plus souhaitables entre producteurs; sur elles seules ne peut reposer la défense des intérêts généraux de l'économie nationale que l'acuité de la concurrence extérieure menace trop souvent de compromettre. "

Sources : Bulletin Quotidien $n^{\circ} 274$ du 8 déc. 1932

Les Ententes Industrielles obligatoires (1935)

Au cour des années trente, les politiques publiques en matière d'ententes économiques deviennent plus active, avec une volonté d'ingérence et de contrôle public. Alors qu'en Allemagne, le gouvernement nazi a rendu, dès son entrée en fonction, la cartellisation - c'est-à-dire la formation d'ententes sectorielles des producteurs et des vendeurs - obligatoire (loi du 15 juillet 1933), en France, face à la crise qui se prolonge, les gouvernements envisagent aussi de mettre en place une telle législation. Celle-ci est une réelle atteinte au libéralisme économique et pourtant les industriels n'y sont pas vraiment opposés, même si, pour d'autres raisons, ils critiquent le projet de loi en discussion au début de l'année 1935.

" Au cours des échanges de vues, auxquels notre Conseil a procédé sur ces questions, il était apparu que si une intervention abusive de l'État dans les affaires privées était unanimement réprouvée, certaines industries particulièrement éprouvées par la crise demandaient de pouvoir contraindre des dissidents à se plier à des accords jugés indispensables par la majorité des entreprises.

Notre Conseil a été ainsi amené à élaborer un projet créant un Comité d'arbitrage qui aurait pu 
déclarer une entente obligatoire pour l'ensemble d'une profession. Composé de représentants d'organisations professionnelles et d'un haut magistrat, le Comité n'aurait statué qu'après s'être assuré qu'une majorité massive était réunie en faveur de l'accord et qu'une adhésion unanime à celui-ci n'avait pu être obtenue d'une conciliation volontaire.

Conscient cependant de la gravité de l'atteinte ainsi portée aux principes de libéralisme économique auxquels il est attaché, notre Conseil avait en même temps demandé que son application fût, au moins au début, limitée à deux des industries particulièrement atteintes.

Le projet du Gouvernement s'est écarté notablement de notre conception. Un premier stade de conciliation n'est pas prévu; le Comité d'arbitrage n'a plus qu'un rôle consultatif et le Gouvernement prononce en dernier ressort. Enfin la reconnaissance du caractère obligatoire de l'entente peut être subordonnée à l'acceptation par la profession de certaines mesures d'ordre social dont l'application onéreuse ajouterait encore aux difficultés que traversent les entreprises.

Les Commissions de la Chambre des Députés, puis de l'Assemblée elle-même, ont aggravé le projet dans nombre de ses dispositions. De nouvelles personnalités paraissant peu qualifiées ont été introduit dans le Comité d'arbitrage. Des mesures de publicité incompatibles avec les conditions d'exploitation des entreprises, ont été introduites. Les clauses d'ordre social ont été développées. Ainsi comme contrepartie, comme rançon, dirions-nous, de l'aide apportée par la puissance publique à une industrie en péril, des sujétions, des charges nouvelles seraient imposées : qui ne voit la contradiction existant entre ces deux préoccupations!

En voulant protéger la main-d'œuvre, on empêchera la réalisation d'accords destinés justement à garantir les occupations des travailleurs en permettant aux entreprises qui les emploient de subsister. Ces observations présentées à la Chambre ne l'ont pas convaincue. Il convient d'espérer que le Sénat apportera au projet les rectifications nécessaires. S'il en était autrement, il n'est pas douteux que des industries, qui seraient disposées à se prévaloir de la loi y renonceraient dans l'impossibilité où elles seraient d'accepter des obligations incompatibles avec le fonctionnement de leurs entreprises. "

Sources : Bulletin Quotidien du 29 mars 1935 , supplément (Assemblée générale de la CGPF du 29 mars 1935)
La position des patrons français sur le problème des ententes internationales, aprèsguerre (1946)

Dans le premier après-guerre, en particulier à cause de l'importante pression des Américains qui souhaiteraient voir s'appliquer à l'économie européenne leur propre législation anti-trust mais aussi en raison des difficultés de la reconstruction, le contexte n'est pas du tout favorable à la reconstitution des ententes internationales. Pourtant les patrons français, et parmi eux les sidérurgistes qui fonctionnaient avant la guerre sur un système complexe d'ententes (comptoirs pour le marché intérieur et pour l'exportation, Cartel international de l'acier et de nombreux autres cartels sectoriels) sont favorables à leur reconstitution. Albert Bureau, le directeur de la sidérurgie, au ministère de l'industrie, consulte, au début de l'année 1946, les sidérurgistes sur ce problème et sur la participation éventuelle de la Chambre Syndicale de la Sidérurgie Française (CSSF). Dès cette date, les sidérurgistes français envisagent de façon inexorable la reconstitution d'une entente internationale de l'acier, d'initiative privée mais avec l'assentiment des gouvernements des pays concernés. Convaincus de l'utilité économique d'un système qui permet d'éviter les aléas de l'anarchie du marché, ce dont la France a tiré bénéfice au temps de l'E.I.A., et qui, selon eux, évite la mise en place de protections douanières excessives, ils espèrent convaincre les Américains qui demeurent rétifs à tout système de cartels ou d'accords restreignant la libre-concurrence. Si dans l'immédiat, ils pensent qu'il est un peu tôt pour envisager, il n'y a aucune raison politique à cela mais uniquement la volonté d'aborder les négociations avec une sidérurgie française revenue à un niveau de production plus favorable. Dès cette date, il n'y a pas réticence vis-à-vis d'une entente avec l'Allemagne; au contraire celle-ci semble aller de soi puisque l'on prend comme référence l'EIA qui était basée sur le couple franco-allemand et que l'on considère que les pays de l'Ouest européen sont "naturellement solidaires".

"Vous avez bien voulu nous demander dans quelle mesure et à quelles conditions l'industrie sidérurgique serait favorable à la reconstitution d'ententes internationales dans lesquelles elle aurait, comme avant guerre dans l'E.I.A., une participation active. Nous pensons qu'une telle participation pose à la fois une question de principe et un problème d'opportunité.

Sur le principe, nous estimons que les arguments qui ont milité dans le passé en faveur de la constitution d'une entente internationale de l'acier sont encore valables aujourd'hui. Certes, l'E.I.A., 
comme la plupart des ententes analogues, est née d'une crise, mais la perspective, dans un délai qui peut-être assez court, d'une surproduction mondiale de l'acier, capable d'engendrer une crise semblable à celle qui se dessinait dès 1926 et qui s'est aggravée brutalement en 1931, ne nous paraît pas la seule raison qui commande la reconstitution d'une entente internationale. Nous considérons l'entente comme un moyen permanent d'organisation et de répartition des marchés. Son rôle est précisément, en période de prospérité, de prévenir les accidents futurs et de promouvoir entre ses participants des relations et des accords propres à réaliser l'adaptation constante de la production et de prix aux besoins de la consommation.

On a adressé aux ententes des reproches que l'on adresse habituellement à tous les monopoles. Nous pensons que ces reproches, d'une manière générale et plus particulièrement en ce qui concerne l'Entente Internationale de l'Acier sont parfaitement injustifiés. En effet, l'entente ne constitue jamais pour les producteurs qui en font partie, un monopole absolu. Il existe toujours en dehors d'elle des dissidents et dans son sein même la dissidence est en puissance et se révèle automatiquement si l'entente prétend imposer à ses membres une politique de prix ou de restriction à la production qui ne tienne pas compte des possibilités internes de la concurrence et des exigences des progrès techniques. L'expérience de l'entente internationale de l'acier est, à cet égard, significative. On rappelle qu'avant guerre, cette entente ne groupait que $30 \%$ des producteurs mondiaux. Pouvait-on prétendre, dans ces conditions, que le jeu de la concurrence eut été éliminé du marché international de l'acier?

Du point de vue français, il est incontestable que L'EIA a permis à l'industrie sidérurgique française de vivre dans des conditions beaucoup plus favorables que celles où elle se serait trouvée si elle avait dû s'exposer au danger d'une concurrence anarchique. D'une part, en effet, l'entente a garanti aux producteurs français la sécurité de leurs débouchés intérieurs que toutes sortes de dumping directs ou indirects des producteurs étrangers menaçaient dangereusement. Elle a permis de maintenir un important courant d'exportations sur les marchés extérieurs. Il est constant que la sidérurgie française, grâce à l'Entente Internationale de l'Acier, a beaucoup mieux maintenu ses positions sur les marchés d'exportation que la plupart des grandes industries françaises exportatrices durant les années de crise.

D'autre part l'entente a permis d'écarter, pour la quasi-totalité des produits sidérurgiques, la pratique de moyens de protections douanières discriminatoires et d'un maniement difficile - droits de douane prohibitifs, taxes anti-dumping, contingentement, etc. - auxquels le gouvernement était obligé d'avoir recours pour assurer la protection de la plupart des autres secteurs d'activité de l'industrie et de la l'agriculture française. C'est en effet un des mérites essentiels de l'entente qu'elle permet, dans le cadre d'accords librement négociés par les intéressés, d'éviter une protection douanière excessive.

Sans doute, une entente internationale ne doit jamais être fixée définitivement dans sa forme et dans son contenu. Les conditions de production d'un pays à l'autre peuvent se modifier, soit en raison de facteurs extérieurs -évolution des prix et de la monnaie, des salaires - soit en raison de la volonté des industriels eux-mêmes et de leurs gouvernements de développer certaines installations, d'orienter dans un sens nouveau telle ou telle fabrication. L'entente doit par conséquences faire l'objet d'ajustements fréquents. Les obligations qu'elle comporte doivent être d'autant plus rigides que la crise est plus sévère, d'autant plus souples que la conjoncture est plus favorable. Nous considérons qu'elle n'en doit pas moins demeurer comme un cadre permanent à l'intérieur duquel les producteurs des pays participants peuvent harmoniser leur action, rationaliser leur production, confronter leurs points de vue.

Un tel programme nous paraît répondre aux préoccupations actuelles qui sont d'insérer au mieux de nos intérêts, et dans des conditions qui suppriment d'avance certains excès possibles de la concurrence, l'industrie sidérurgie française dans le complexe des pays producteurs de l'ouest européen, naturellement solidaires en raison de leurs conditions respectives d'approvisionnement, de transport et de main-d'œuvre.

Mais il serait vain d'attendre de l'entente internationale une efficacité réelle si l'on prétendait lui retirer certains des caractères qui participent de son essence même et la priver pratiquement de ses moyens d'action. Nous avons dit que l'entente devait évoluer en fonction des circonstances et que sa réglementation devait être d'autant plus rigide que la crise était plus grave. C'est pourquoi il ne saurait être question dans les engagements que pourraient être amenés à prendre nos négociateurs, de renoncer par avance à toute clause réservant aux participants de l'entente leurs marchés nationaux. De même le propice de la répartition, jusqu'à concurrence d'un certain quantum, des marchés extérieurs où il n'existe pas d'industries sidérurgiques, ne saurait être abandonné.

Nous pensons, au surplus, que les abus, que certains semblent redouter, peuvent être plus facilement éliminés par l'adoption d'un statut 
international des ententes qui donne à tous les gouvernements intéressés et aux consommateurs, des garanties suffisantes. A cet égard, s'il nous apparaît nécessaire, pour des raisons techniques et pratiques, et dans l'intérêt même des gouvernements soucieux de conserver leur entière liberté d'action dans les négociations internationales, de laisser aux ententes un caractère privé, nous pensons que cette condition n'exclut nullement certains contrôles, comme ceux qui résulteraient de l'enregistrement obligatoire des ententes par chaque industrie participante auprès de son gouvernement, ainsi que du fonctionnement d'un organisme international chargé de recueillir les plaintes formulées à l'égard des ententes et de faire en conséquence les recommandations nécessaires.

$\mathrm{Au}$ surplus il est hors de doute qu'une entente aussi importante que celle de l'acier ne pourrait être utilement négociée et conclue par l'industrie sidérurgique française sans l'assentiment et l'appui des pouvoirs publics. Toutes les négociations qui ont été menées avant la guerre dans le cadre de l'entente internationale de l'acier par les industriels français, l'ont toujours été au vu et au su de leur gouvernement.

Notre position nous paraissant ainsi, par le principe, clairement définie, il reste à examiner si la reconstitution d'une entente internationale de l'acier serait actuellement opportune. Nous répondons par la négative. En effet, le chemin qui doit y conduire doit être jalonné au préalable par un certain nombre de consultations et d'échanges de vues, et aussi par des progrès suffisants de l'activité de nos usines et de leurs potentiels d'exportation. Nous ne saurions en effet, nous présenter dans les futures négociations internationales sans nous être constitué des références qui nous permettent d'obtenir sur le marchés extérieurs contrôlés par l'entente une place conforme aux possibilités de notre industrie, lorsque celle-ci aura au moins entamé la réalisation de son programme de rééquipement et sera libérée des restrictions qui pèsent actuellement sur ses approvisionnements.

Il n'en est pas moins indispensable de fixer, dès maintenant notre attitude et nous pensons qu'en assortissant les statuts nouveaux des ententes internationales de clauses qui leur assurent une souplesse suffisante et qui ouvrent contre les abus possibles certaines procédures de recours, on doit pouvoir faire admettre au gouvernement américain que les ententes, loin de constituer une entrave au commerce international et à la libre concurrence, sont des moyens essentiels de réorganisation des marchés et d'adaptation mutuelle des productions, sans lesquelles la concurrence serait génératrice d'anarchie et conduirait inévitablement à une nouvelle fragmentation du monde en systèmes économiques fermés. "

Sources: Archives Nationales 62 AS 99/ CSSF

lettre datée du 8 mars 1946, de Jules Aubrun au

Directeur de la Sidérurgie.

\section{Le problème de la Ruhr vu par le patronat français(1948)}

Au vingtième siècle, la Ruhr forme, avec la Sarre, les Ardennes belges et luxembourgeoises et la Lorraine, le cour de l'Europe industrielle. Ces régions ont été parmi les enjeux des deux guerres mondiales et sont également un de ceux de l'aprèsguerre. Le charbon de la Ruhr est indispensable à l'industrie lourde européenne, a fortiori aux industriels français. Mais la puissance industrielle concentrée dans la Ruhr, prestigieux symbole de la puissance $d u$ Reich, fait peur à ses voisins européens, avant tout pour des raisons sécuritaires mais aussi pour des raisons économiques. Avec la défaite allemande, la tentation a été grande parmi les Alliés - et particulièrement en France - d'en profiter pour anéantir à la fois la menace et la concurrence allemande. Mais les Américains, en apportant l'aide Marshall à tous les pays d'Europe occidentale, Allemagne comprise, en ont décidé autrement pour l'avenir économique de l'Europe. Si les gouvernements français ont encore du mal à intégrer cette donnée nouvelle, pour les industriels, qui déjà construisent l'Europe en créant le Conseil des fédérations industrielles d'Europe (CIFE) en 1948, et en y intégrant l'Allemagne, l'urgence n'est plus aux tergiversations mais au réalisme économique.

" ( ..) L'opinion française a certainement été émue par les décisions bilatérales prises par les généraux Clay et Robertson, avec l'accord de leur gouvernement, à l'égard du régime futur de la Ruhr; Le président de la République a cru bon d'élever une protestation, qui ne rentre peut-être pas très exactement dans son rôle constitutionnel - mais il a coutume d'en sortir- et qui n'étaient pas conçue en termes adéquats à notre position internationale, bien qu'il connaisse celle-ci mieux que personne, par les responsabilités qu'il a assumées à titres divers mais d'une manière continue depuis la libération.

Mais regardons surtout vers l'avenir: que s'agit-il pour nous d'obtenir dans la Ruhr? Les gens pratiques répondent du charbon. Mais ce sont des mystiques qui nous gouvernent. Fi de ces réalités! Nous réclamons, parait-il, un contrôle international de la Ruhr. Bravo! Nous y appellerons sans doute nos bons amis les Soviets. Le contrôle à quatre à Berlin donne de si beaux résultats que l'on 
comprend sans peine que nous éprouvions le besoin de l'étendre à la Ruhr. Car de quel droit expulserait-on les Russes du contrôle international de la Ruhr, si quelque jour il prenait vie?

Eh quoi! objecte le chef de l'État lui-même, vous n'y comprenez rien: rendre la Ruhr aux Allemands c'est reconstituer le potentiel de guerre d'un futur Reich, qui reprendra une fois encore, la politique d'hégémonie européenne dont la France a déjà fait trois fois les frais! Le risque est en effet certain. La question, toutefois, est posée des meilleures méthodes pour réduire ce risque; Du contrôle et de son efficacité, nous avons déjà fait l'expérience. On peut la considérer comme décisive. Le conseil n'aboutit à rien, si ce n'est à envenimer le rapport entre les contrôleurs et le contrôlé. Ajoutant que, possible tant que la force de l'occupant en garantit l'exercice, il devient pratiquement irréalisable quand cesse l'occupation. Qu'à cela ne tienne! répondent nos rêveurs impénitents : il faut occuper l'Allemagne pendant au moins 40 ans, et la Ruhr, définitivement. Ce programme a fort belle allure et peut séduire les passionnés et les ignorants. Mais les raisonnables et les avertis demanderont: êtesvous sûrs que les Américains et les Anglais occuperont l'Allemagne aussi longtemps ? Et comment occuperez-vous la Ruhr, isolée en terre allemande, si vous n'avez pas d'autre communication avec elle qu'un nouveau pont aérien? Et le ferez-vous enfin contre la volonté des Américains et des Anglais? Nous avons déjà fait une expérience d'occupation dans la Ruhr, du temps de M. Poincaré. On ne saurait dire qu'elle ait réussi.

Au surplus, à quoi bon s'attarder dans une attitude à laquelle nos alliés eux-mêmes sont fondés à croire que nous avons explicitement renoncé lorsque nous avons adhéré au programme de reconstruction européenne? Tous les commentateurs du plan Marshall, à l'époque, nous ont avertis, non pas à une mais à maintes reprisesque la participation de l'Allemagne était une condition essentielle du relèvement de l'Europe. Nous n'avons fait aucune réserve sur ce sujet, tant nous avions besoin de l'aide américaine pour nous prémunir contre toutes les faillites accumulées par les gouvernements de la $\mathrm{IV}^{\circ}$ république. Bien mieux, il est de notoriété publique que les ÉtatsUnis souhaitaient voir la France prendre la direction de la reconstruction européenne, qu'ils désiraient une Union européenne animée par la France. Oui, ce sera bien l'écrasante responsabilité des nains de la Libération d'avoir gaspillé cette chance unique d'une prépondérance française dans les États-Unis d'Europe. (...)

[Si l'État avait chassé le communisme et encouragé la libre entreprise], nous alignerions des résultats très supérieurs à ceux que, déjà, l'initiative privée a su conquérir, en dépit de l'incapacité de nos mauvais gérants. Et nous ne risquerions pas de voir, demain, l'Allemagne prendre notre place dans l'union européenne. Un enfant de 7 ans comprendrait que nous n'obtiendrons pas le charbon de la Ruhr pour faire de l'acier français, quand les mines, les hauts fourneaux et les aciéries allemandes sont prêtes à travailler à plein et que les nôtres sont, périodiquement, paralysées sur l'ordre du Kominform !

Quand nous nous révélons incapables d'assurer, même avec l'appui des crédits américains, l'équilibre de notre propre économie et de nos propres finances, sommes nous qualifiés pour réclamer la direction de la reconstruction européenne ? Et laisserons-nous dire, dans le monde entier, que nous voulons freiner la production allemande parce que nous ne réussissons pas à organiser et à activer la nôtre ? ( ...)

La vérité, que nous avons souvent proclamée, est que la $\mathrm{IV}^{\circ}$ république issue de l'imposture et de l'usurpation, est incapable de grande politique à l'extérieur comme à l'intérieur. Rendons grâce à $\mathrm{M}$. Queuille et Schuman d'avoir appelé M. FrançoisPoncet à l'activité. Peut-être grâce à lui, auronsnous demain une ligne de conduite qui s'inspire à la fois des intérêts permanents de la France et des impératifs actuels de la collaboration francoaméricaine et européenne. Nous avons déjà perdu une importante bataille économique en Europe. Il s'agit de ne pas perdre la bataille politique. (...)" Sources: Archives Nationales 72 AS / 356 hebdomadaire "le Bulletin " (de France Documents) $d u$ 19 nov. 1948, article "Discourir n'est pas agir", non signé

\section{Inquiétudes des sidérurgistes français face au futur traité de la CECA (1951)}

Lorsque le 9 mai 1950, Robert Schuman lance une offre solennelle à l'Allemagne de mise en commun du charbon et de l'acier des deux pays, offre ouverte aux autres pays européens qui en émettrait le vou, les sidérurgistes français soutiennent dans un premier temps cette initiative courageuse et tournée vers l'avenir. Mais rapidement le désenchantement grandit à mesure que se déroulent les négociations pour la préparation $d u$ traité de la Communauté Européenne du Charbon et de l'Acier. Au début de l'année 1951, le conflit est ouvert.

" Nous manquerions à notre devoir si nous n'attirions pas solennellement votre haute attention, 
et l'attention du gouvernement français, sur les conséquences désastreuses et irrémédiables qu'aurait pour notre industrie et pour l'économie française tout entière le traité en préparation.

S'il exposait les entreprises sidérurgiques françaises à une concurrence inégale de la part des producteurs étrangers jouissant d'avantages exceptionnels, du fait notamment de leurs ressources financières (alors que la sidérurgie française est gravement débilitée par les pertes qu'elle a subies, de par l'insuffisance des prix fixés sur le marché international, et les conditions désastreuses dans lesquelles elle a dû prendre des commandes à l'exportation pour maintenir son taux de marche et d'emploi dans une période où l'exportation était largement déficitaire et le marché intérieur défaillant), ou de leur liaison avec des charbonnages (alors qu'en France la nationalisation des houillères rend radicalement impossible aucune connexion semblable).

S'il comprenait les ressources en minerai de fer de Lorraine, ressources d'importance très limitée (et dont l'épuisement ôterait toute raison d'être, et toute possibilité de survie aux usines sidérurgies Lorraines) ou en faisant, ou en risquant d'en faire, d'une manière ou de l'autre, le bien commun de l'ensemble des industries sidérurgiques du pool alors que les principales industries concurrentes de l'industrie française, à l'intérieur du pool, ont par leur situation géographique, la facilité que n'a pas la sidérurgie Lorraine de recevoir à bon compte des minerais extérieurs.

S'il soumettait, dans l'organisation de ce marché, la sidérurgie française au pouvoir réglementaire et financier d'une autorité internationale, dont les tendances sont a priori, inconnues, sans possibilités suffisantes de recours et sans que le gouvernement français pût, s'il le juge utile, efficacement intervenir pour éviter que cette action ne l'étouffa ou la conduisit à la ruine.

La vie de notre industrie est en cause; elle est en cause non seulement pour l'avenir immédiat, mais pour un demi-siècle. La sidérurgie est un des fondements de l'économie française. Elle est l'un des outils essentiels de l'équipement et de la défense de la France."

Sources: Archives Nationales 62 AS 113 lettre de Charvet à Schuman, du 19 mars 1951.

\section{La sidérurgie française et le pool charbon- acier}

Alors que les réactions négatives avaient été vives, tant du côté des industriels allemands que français, lors de la signature du traité de la CECA (18 avril 1951), dès que la Communauté installe ses institutions et que celles-ci commencent à fonctionner, ces mêmes industriels basculent dans une attitude nettement positive mais sans dissimuler leurs craintes pour l'avenir.

" Voilà quatre mois que la Haute Autorité s'est installée à Luxembourg; c'est évidemment peu de chose sur les cinquante ans que doit durer le Plan Schuman. C'est également une période préliminaire à l'ouverture du marché unique, puisque je vous rappelle que les dates fatidiques seront, en 1953, le 10 février: ouverture du marché commun pour le charbon, le minerai de fer et la ferraille, puis le 10 avril pour l'acier. C'est à l'ouverture de ces marchés uniques que l'on pourra commencer à porter un jugement sur les modifications plus ou moins profondes que la mise en oeuvre du Plan Schuman apportera dans l'économie de l'industrie lourde européenne. Mais tout de même, ces quatre mois ont été bien remplis, et peut-être serez-vous intéressés de connaître une impression à ce sujet.

Et d'abord, quid de la Haute Autorité? Eh bien je dis très franchement, nous sommes en France très heureusement impressionnés par la façon dont la Haute Autorité de Luxembourg, sous la présidence de M. Jean Monnet, a pris possession de ses fonctions, a installé et est en train d'installer ses services, a pris des contacts avec les professionnels et commencé à définir les grandes lignes de sa politique. Nous sommes vraiment très heureux de voir avec quel sens aigu et très réaliste de leurs responsabilités, qui sont évidemment formidables, M. Jean Monnet et ses collègues ont pris en main les destinées du Traité de la Communauté. Nous avons pu craindre un moment, pendant toute la période d'élaboration du Traité - je le dis bien franchement - que l'application de ce Traité reflétât peut-être plus de volonté doctrinale que de sens des réalités économiques. Nous sommes très heureux de voir que toute l'orientation qui est donnée depuis quatre mois à son organisation, à la mise en place de ses Commissions de travail, à ses contacts avec l'Angleterre, les États-Unis, nous sommes très heureux de voir que tout cela part d'un sens des réalités et d'un sens politique et économique très opportun des difficultés inévitables et considérables auxquelles risque de se heurter au départ un phénomène de mutation aussi brusque que cette mise en communication de six économies très différentes. 
Nous avons relevé avec beaucoup d'intérêt et beaucoup de satisfaction la volonté qui a été manifestée à maintes reprises par M. Jean Monnet d'élaborer tout cela en commun avec les États et avec les professionnels. Il a constitué autour de lui toute une série de commissions de travail, dans lesquelles les industriels du charbon et de l'acier des six pays sont représentés. (...) Monnet a donné les assurances que toutes les précautions seront prises là où l'ouverture du marché commun pourrait créer des désordres graves faute de préparation suffisante, pour ménager les transitions; en particulier, notre Gouvernement a obtenu tout récemment des apaisements sur un point qui nous préoccupait beaucoup et qui était celui de l'ouverture du marché unique de la ferraille. Nous sommes, d'autre part, satisfaits de la représentation qui a été donnée à nos industries dans les Commissions de travail. Il y a bien quelques petites retouches à faire par-ci, par-là, mais l'expérience a montré que ces choses-là se réglaient très facilement et dans un esprit très réaliste. Nous sommes également très satisfaits des nominations auxquelles le Conseil des Ministres de la Communauté vient de procéder en ce qui concerne le Comité consultatif qui a été créé, comme vous le savez, la semaine dernière. Les désignations des représentants français sont aussi satisfaisantes que possible.

Enfin, nous devons nous féliciter du choix des techniciens français qui ont été appelés par M. Jean Monnet dans son administration. Nous avons été vraiment très heureux de voir que le Président de la Haute Autorité avait choisi les meilleurs de nos techniciens de la sidérurgie pour leur confier des responsabilités importantes. Tout ceci ne peut que renforcer les déclarations que je faisais au début du mois de juillet, mais que je faisais comme un acte de foi, et que j'ai faites en faisant confiance à la sagesse et au sens politique de la Haute Autorité. Je tiens à dire que la Haute Autorité, après ces quatre mois d'expérience que nous avons eus, peut vraiment compter sur la collaboration la plus loyale de la Sidérurgie française.. (...)

[Il rend hommage au travail de Jean-Marie Louvel, ministre de l'industrie désigné comme ministre Charbon-Acier dans le Conseil des ministres du Plan Schuman et le remercie de son sens de la collaboration avec les milieux professionnels ou syndicalistes concernés. Il remercie amicalement l'administration $d u$ ministère de l'industrie avec laquelle la sidérurgie travaille depuis longtemps "la main dans la main", et pour finir Robert Schuman et le nouveau commissaire au Plan. Il se félicite enfin de la confirmation du rattachement économique de la Sarre à la France.]
Enfin, pour achever ce palmarès de nos motifs de remerciements, je dirai que nos relations avec les Charbonnages de France sont aussi étroites que possible. Bien sûr, nous aurons de temps en temps des intérêts qui seront divergents, mais nous avons décidé de conclure un pacte défensif et offensif et c'est vraiment cet accord étroit entre les grandes industries nationalisées et la grande industrie privée que nous sommes, qui est le trait caractéristique d'une collaboration dont je dirai tout à l'heure que je souhaiterais la voir étendue à tout l'ensemble de la collectivité française.

Je ne peux pas dissimuler que j'ai quelques désillusions du côté de certains membres du Gouvernement. J'imaginais avec un peu de candeur que, nous demandant de partir en avant-garde sur une route semée de chausse-trapes et propice à des coups d'arquebuses, l'industrie du charbon et de l'acier bénéficierait d'une espèce de préjugé favorable de la part du Gouvernement et de la part de l'ensemble de l'administration française (...). D'ailleurs, on nous l'avait promis et on n'a qu'à se reporter à ce qu'étaient les débats de l'Assemblée nationale et du Sénat lors du vote de la Loi de ratification. Le Parlement a pris acte de la nécessité d'une vigoureuse politique d'investissements dans les Charbonnages et dans la Sidérurgie. La promesse d'une harmonisation tout à fait substantielle de nos charges fiscales et de nos charges financières nous avait été faite pour nous permettre d'être en état de compétition avec nos principaux concurrents.

Maintenant, on me dit: le Plan Schuman, le Budget, la Trésorerie, le Parlement..., ce qui d'ailleurs correspond à une situation qui est ce qu'elle est et dont je ne devrais pas m'étonner, mais dont je m'inquiète. La vérité c'est que j'ai le sentiment qu'après avoir tiré de très beaux feux d'artifice à la tribune du Parlement au moment de la ratification, on ne pense plus beaucoup au Plan Schuman en dehors d'un ou deux ministres spécialisés ou d'une ou deux administrations spécialisées. (...) On nous avait promis ces investissements, mais vous savez ce que sont les nécessités budgétaires. Au total, en ce qui concerne la Sidérurgie, on nous a accordé, dans des conditions qui sont tout à fait méritoires, car il n'y a pas grand-chose à partager, 37 milliards l'année prochaine, là où d'après les services il en eut fallu 60. (...) Nous attendons encore que les pouvoirs publics nous donnent les moyens qui doivent nous permettre de réaliser dans des conditions satisfaisantes les opérations de financement indispensables pour compléter les crédits très insuffisants qui nous sont accordés par ailleurs. Charges financières: nous avons obtenu quelque chose, cela correspond à la moitié de ce que la 
Commission des finances avait recommandé.

Je sais bien qu'on m'oppose l'orthodoxie financière, à quoi je réponds que le Plan Schuman va obliger à écrire des chapitres absolument inédits et sans précédent aucun dans l'histoire du droit français, des chapitres inédits de droit constitutionnel, de droit international, de législation fiscale, sociale et financière. Et je suis un peu fâché qu'on m'oppose des précédents au nom d'une législation très valable pour une industrie qui reste dans le droit commun, mais que je conteste quand on pense les opposer à celle que nous représentons. (...)

Alors tout ceci tire son actualité des inquiétudes vraiment assez préoccupantes que nous avons, sans même parler de ce qui se passera dans les cinquante ans à venir, sur les premières conséquences de l'ouverture du marché unique. On peut bien dire qu'à l'ouverture du marché unique le 10 février, nous perdons à peu près, nous Français, sur tous les tableaux.(...) Nous risquons de perdre sur le minerai de fer et la ferraille parce que, jusqu'à présent, nos ressources en minerai de fer et ferrailles étaient réservées à la France par un régime de prohibition d'exportations ou de contrôle au compte-gouttes des exportations. Le marché unique établissant des vases communicants, il est certain que la Belgique, le Luxembourg, l'Allemagne, en ce qui concerne le minerai, les mêmes plus l'Italie en ce qui concerne la ferraille, seront enclins à se tourner vers les ressources françaises, ceci se traduisant par des hausses de prix de nos matières et par une diminution de nos approvisionnements. C'est vraiment un point préoccupant. Je doute qu'il y ait grand-chose à faire pour le minerai. Pour la ferraille, la situation serait tellement dramatique que nous espérons que certaines mesures qui ne seront que temporaires seront prises pour ménager une transition, mais ce ne sera qu'une transition.

Au mois d'avril, s'ouvrira le marché unique de l'acier. Il est certain que là nous démarrons aussi avec un handicap. Toutes les sociétés sidérurgiques vont aborder le marché unique de l'acier avec des trésoreries extraordinairement serrées, rendues telles par un régime de fixation étroite des prix de l'acier dans le passé, et par le sacrifice, que nous avons consenti au mois de mai dernier, d'une baisse de prix représentant 10 milliards de francs en faveur de l'expérience de M. Pinay. Ces 10 milliards nous manquent. Dans le même temps, les Allemands, avec un sens étonnant des impératifs du Plan Schuman, au mois d'août, huit jours avant l'installation à Luxembourg de la Haute Autorité, mettaient en liberté le prix de l'acier. La Sidérurgie allemande a augmenté à quatre reprises le prix de l'acier depuis le mois d'août, ce qui nous donne la satisfaction de voir que le prix de l'acier allemand est supérieur de 10 à $15 \%$ à celui de France, mais cette augmentation n'a d'autre intérêt que de lui donner une marge extraordinairement copieuse d'autofinancement pendant la période qui précède l'ouverture du marché unique. La Sidérurgie allemande va aborder le marché unique avec des trésoreries opulentes, notre Sidérurgie et nos Charbonnages de France, avec des trésoreries extrêmement étroites: c'est une mauvaise condition au départ. (...)

En ce qui concerne la production, nous sommes vraiment pleins d'optimisme. Nous avons, au mois d'octobre, atteint le record de la production d'acier: 1 million de tonnes d'acier brut en France. (...) Ce qui nous inquiète, c'est évidemment de constater l'affaissement de nos carnets de commandes, le marasme qui règne dans beaucoup de secteurs de l'industrie qui sont nos clients, le véritable drame de notre commerce extérieur aussi bien de nos exportations directes que de nos exportations indirectes, et il est bien évident que c'est un acte de foi que de pousser à fond notre production alors que nous avons des motifs assez sérieux d'inquiétude.

Messieurs, ceci pour conclure. Il s'est tenu à Trèves, il y a un mois, une grande réunion qui avait été organisée par le Patronat allemand et qui rassemblait huit cents personnes. Il y avait les deux Ministres allemands, tous les hauts personnages de l'administration allemande, de l'industrie de transformation allemande, les sidérurgistes, les charbonniers, les représentants des syndicats ouvriers. Ils avaient invité: M. Spaak, en sa qualité de Président de l'Assemblée du Plan Schuman, M. Monnet qui s'était fait représenter. Les Français qui assistaient à cette manifestation sont revenus vraiment très impressionnés par l'extraordinaire cohésion avec laquelle l'Allemagne abordait le Plan Schuman, par cette espèce de volonté unanime où toutes les forces vives du pays se serraient dans un faisceau pour gagner une partie dont vous savez que l'enjeu est considérable et dépasse de beaucoup le cadre du charbon et de l'acier. Le voeu que j'exprime, c'est que la France veuille réaliser quelque chose du même ordre, c'est que, dans tous les milieux, on prenne le Plan Schuman au sérieux.

En ce qui nous concerne, nous faisons notre possible pour nous préparer à ce combat pacifique, mais ce combat a un enjeu énorme. Il reste à obtenir que l'ensemble du Pays, l'ensemble du Parlement et le Gouvernement prennent conscience de cette solidarité indispensable. Ils en prendront conscience dans la mesure où vous le leur direz, dans la mesure où vous leur direz que c'était très beau pour la France de tenir le flambeau qui illumine la route qui nous conduit à l'intégration 
européenne, mais que ce serait un drame si l'intégration se faisait sur le cadavre de l'industrie française. Il n'y a aucune espèce de raison d'être défaitiste. Nous avons des atouts magnifiques. Mais les problèmes sérieux, nous devons les étudier sérieusement avec la volonté de mettre tout en oeuvre pour les résoudre au mieux. "

Bulletin du CNPF $n^{\circ} 91$ (janvier 1953) Allocution prononcée devant la presse par M. Pierre Ricard, le 10 décembre 1952

\section{La construction de l'Europe}

Très vite, l'entrée en application du Plan Schuman laisse entrevoir un élargissement rapide à d'autres secteurs de cette communauté économique européenne en formation. Les patrons français et européens sont bien décidés à participer activement à cette construction, par conviction mais aussi afin de garantir leurs intérêts.

" La construction de l'Europe est maintenant une réalité. Non seulement le plan Schuman entre en application, mais on se rend compte dès maintenant que le cadre initial peut être très vite débordé, d'abord vers les industries en amont qui fournissent leurs équipements aux charbonnages et à la sidérurgie; ensuite, vers l'ensemble des industries transformatrices en aval. Les conséquences économiques de la communauté de défense projetée ne seraient pas moins importantes. Et, sur un plan tout à fait général, les récents travaux de Strasbourg font apparaître l'éventualité d'une constitution européenne comme une probabilité à assez court terme.

L'idée européenne, au principe de laquelle vous avez, d'ailleurs, eu l'occasion de marquer votre faveur, n'est donc plus maintenant à discuter; et c'est aux conditions de sa réalisation qu'il faut être extrêmement attentif. Les problèmes qu'elle pose, tel celui des répercussions de l'intégration européenne sur la structure de l'Union française sont, en effet, de la plus haute gravité.

Dans les années qui viennent, vont être prises des décisions essentielles qui engageront l'avenir de l'Europe. Il faut que le C.N.P.F. et nos professions se tiennent très au courant de ces problèmes et étudient sérieusement les conséquences des solutions envisagées, si nous voulons, en particulier, que l'Europe future soit une Europe de libre entreprise.

C'est une des raisons qui nous a amenés, comme vous le savez, à prendre l'initiative de la création du Conseil des Fédérations industrielles d'Europe; l'utilité de cet organisme a été progressivement mieux comprise par tous ses adhérents, parmi lesquels je suis heureux de dire que nos amis anglais semblent maintenant vouloir jouer un rôle particulièrement important. Aussi, la dernière Assemblée générale du C.I.F.E. a-t-elle décidé de lui donner une compétence tout à fait générale. En même temps, elle a prévu la constitution, en son sein, de groupements plus restreints, et, dans ce cadre, il faut certainement attacher une grande importance à la création de l'Union des industries des six pays de la Communauté européenne, sous la présidence de M. Quintieri, représentant de l'Italie. "

Bulletin du CNPF $n^{\circ} 92$ du 20 janvier 1953 (Assemblée générale du 16 janvier 1953) 